\title{
Role of the microRNA-214/Bax axis in the progression of acute liver failure
}

\author{
SHAOHONG WU ${ }^{1,2}$, XIAOPING HUANG $^{1}$, WEI SUN ${ }^{1}$, LI CHEN $^{1}$, YAN HUANG ${ }^{1}$, \\ YAN WANG $^{1}$, ERPING LUO ${ }^{1}$, AILAN QIN ${ }^{1}$, WEIFENG ZHAO ${ }^{1}$ and JIANHE GAN ${ }^{1}$ \\ ${ }^{1}$ Department of Infectious Diseases, The First Affiliated Hospital of Suzhou University, Suzhou, Jiangsu 215006; \\ ${ }^{2}$ Department of Emergency Critical Disease, Shanghai General Hospital, Shanghai 201620, P.R. China
}

Received March 26, 2019; Accepted February 28, 2020

DOI: $10.3892 / \mathrm{mmr} .2020 .11123$

\begin{abstract}
Acute liver failure (ALF) is a fatal liver disease characterized by severe hepatocyte destruction. MicroRNAs (miRNAs/miRs) have been reported to serve a key role in a number of liver diseases. Therefore, the aim of the present study was to investigate the role and underlying mechanism of miR-214 in ALF. ALF murine and hepatocyte models were established using D-galactosamine (D-GalN) and lipopolysaccharide (LPS) or D-GalN + tumor necrosis factor (TNF)- $\alpha$, respectively. The expression levels of miR-214 and Bax were detected by reverse transcription-quantitative polymerase chain reaction (RT-qPCR) and/or western blotting. Furthermore, an automatic biochemical analyzer was used to measure the levels of aspartate aminotransferase (AST) or alanine aminotransferase (ALT). The levels of TNF- $\alpha$ and interleukin (IL)- 6 were detected by ELISA and RT-qPCR. In addition, TUNEL staining and flow cytometry were used to analyze cell apoptosis, and the protein expression of caspase- 3 was determined by western blotting. It was identified that the levels of AST and ALT were increased and that hepatocyte apoptosis was enhanced in the D-GalN/LPS-stimulated group compared with the control. Furthermore, higher expression of caspase-3 was observed in the D-GalN/LPS-stimulated group. In addition, it was demonstrated that miR-214 was downregulated, while Bax was upregulated in D-GalN/LPS-stimulated mice and D-GalN/TNF- $\alpha$-stimulated BNLCL2 cells. Moreover, in D-GalN/TNF- $\alpha$-stimulated BNLCL2 cells, miR-214 overexpression suppressed apoptosis and decreased TNF- $\alpha$ and IL-6 levels, and these effects were reversed by the Bax plasmid. It was also identified that overexpression of miR-214 significantly decreased Bax mRNA and protein expression
\end{abstract}

Correspondence to: Dr Jianhe Gan, Department of Infectious Diseases, The First Affiliated Hospital of Suzhou University, 188 Shizi Street, Suzhou, Jiangsu 215006, P.R. China

E-mail: ganjh295968@163.com

Key words: acute liver failure, microRNA-214, Bax, hepatocytes, apoptosis levels in vitro. Collectively, the present results suggested that miR-214 inhibited hepatocyte apoptosis during ALF development via targeting Bax, thus indicating that miR-214 may be a potential target for ALF treatment.

\section{Introduction}

Acute liver failure (ALF) is a rapidly developing disease, with a rapid onset of symptoms, that is associated with multi-organ failure (1). The main causative factors of ALF include drug toxicity and hepatitis virus infection (2). D-galactosamine (D-GalN) and lipopolysaccharide (LPS) are common biochemical reagents that may be used to establish fulminant hepatic failure (FHF) injury models, which effectively simulate the FHF clinical state (3). During ALF development, one of the key pathological traits is the associated immoderate immune cascade response, which leads to extensive liver cell apoptosis and defective liver cell proliferation (4). However, little is known regarding the mechanism of action of microRNAs (miRNAs) in the process of ALF (5).

miRNAs are a class of endogenous non-coding RNAs, measuring 19-22 nucleotides in length, that regulate the expression of target genes by interacting with the 3 -untranslated (UTR) regions of these genes at the post-transcriptional level (6). Furthermore, miRNAs have been identified as potential biomarkers in the pathological processes of several life-threatening diseases, including ALF (7). Abnormal regulation of miRNAs has also been observed in various liver diseases, and miRNAs serve a key role in regulating hepatocyte proliferation (8) and liver development $(9,10)$. For example, miR-122 is one of the most common miRNAs in the liver, and is involved in appropriate proliferation and differentiation of liver cells (11). In addition, miR-122 has been implicated in viral hepatitis and liver disease (12). Moreover, it has been reported that miR-125b-5p could inhibit ALF (13). It has also been suggested that miR-214 exerts anti-fibrotic effects in chemically induced liver fibrosis and cirrhosis (14). However, the role of miR-214 in human ALF remains unknown.

Therefore, the aims of the present study were to investigate the role of miR-214 in ALF and to elucidate its mechanism of action. 


\section{Materials and methods}

Experimental animals and study design. A total of 30 male BALB/c mice (age, 6-8 weeks; weight, 20-22 g) were purchased from Shanghai SLAC Laboratory Animal Co. Ltd., and were housed in a standard animal housing facility (temperature, $22-24^{\circ} \mathrm{C}$; humidity, 60-65\%) with ad libitum access to food and water under a 12-h light/12-h dark cycle. The mice were randomly divided into two groups (control and ALF model groups; $n=15 /$ group). To establish the mouse model of ALF, the mice were administered D-GalN [800 mg/kg body weight intraperitoneal (i.p.); Sigma-Aldrich; Merck KGaA] and LPS (10 $\mu \mathrm{g} / \mathrm{kg}$ body weight, i.p.; Sigma-Aldrich; Merck KGaA) as described previously (15). Mice in the control group were treated with $500 \mu l$ saline by i.p. injection. Mice were anesthetized with pentobarbital $(50 \mathrm{mg} / \mathrm{kg})$ by i.p. injection and sacrificed by cervical dislocation to collect blood samples (1 $\mathrm{ml})$ at $0,1,3,5,7$ and $9 \mathrm{~h}$ after D-GaIN/LPS treatment for aspartate aminotransferase (AST) or alanine aminotransferase (ALT) detection. Animal death was defined as the lack of heartbeat or respiration. The blood $(1 \mathrm{ml})$ of mice at $7 \mathrm{~h}$ after D-GaIN/LPS treatment was collected for interleukin (IL)-6 and tumor necrosis factor (TNF)- $\alpha$ detection.

All animal care and experimental protocols were performed strictly according to the recommendations in the Guide for the Care and Use of Laboratory Animals by the National Institutes of Health and the Animal Ethics Committee of The First Affiliated Hospital of Suzhou University. The present study was approved by the Animal Ethics Committee of The First Affiliated Hospital of Suzhou University. Moreover, there was no mouse mortality during the aforementioned experimental procedures. The experimental end-point was when mice lost $>15 \%$ of their body weight.

Cell culture and treatment. Normal murine embryonic liver cells (BNLCL2) were provided by Wuhan Procell Life Technology Co., Ltd. (https://www.procell.com.cn/view/537. html) and cultured in DMEM (Gibco; Thermo Fisher Scientific, Inc.) supplemented with 10\% FBS (Gibco; Thermo Fisher Scientific, Inc.), $4 \mathrm{mM}$ glutamate and $1 \%$ penicillin/streptomycin (Gibco/Invitrogen; Thermo Fisher Scientific, Inc.) at $37^{\circ} \mathrm{C}$ in a humidified chamber with $5 \% \mathrm{CO}_{2}$.

BNLCL2 cells were treated with $1 \mathrm{mg} / \mathrm{ml} \mathrm{D-GalN}$ (Sigma-Aldrich; Merck KGaA) and $100 \mathrm{ng} / \mathrm{ml} \mathrm{TNF}-\alpha$ (Sigma-Aldrich; Merck KGaA) at $37^{\circ} \mathrm{C}$ for $36 \mathrm{~h}$ to induce the hepatocyte injury model in vitro.

For miR-214 mimic treatment, BNLCL2 cells were transfected with $100 \mathrm{nM}$ mimic control (sense, 5'-UUUGUA CUACACAAAAGUACUG-3' and anti-sense, 5'-CAGUAC UUUUGUGUAGUACAAA-3'; Guangzhou RiboBio Co., Ltd.), $100 \mathrm{nM}$ miR-214 mimic (sense, 5'-ACAGCAGGC ACAGACAGGCAGU-3' and anti-sense, 5'-ACUGCCUGU CUGUGCCUGCUGU-3'; Guangzhou RiboBio Co., Ltd.) or $100 \mathrm{nM}$ miR-214 mimic $+1 \mu \mathrm{g}$ Bax CRISPR activation plasmid (cat no. sc-419292-ACT; Santa Cruz Biotechnology, Inc.) for $24 \mathrm{~h}$ using Lipofectamine ${ }^{\circledR} 2000$ reagent (Invitrogen; Thermo Fisher Scientific, Inc.), according to the manufacturer's protocol. Subsequently, cells were treated with D-GalN $(1 \mathrm{mg} / \mathrm{ml})$ and TNF- $\alpha(100 \mathrm{ng} / \mathrm{ml})$ at $37^{\circ} \mathrm{C}$ for $36 \mathrm{~h}$ and used for further analysis.
Transfection of miR-214 mimic in cells. miRNA mimic is small double-stranded RNA oligonucleotide, which can simulate endogenous mature miRNA molecules (16). The synthesized miR-214 mimic was purchased from Guangzhou RiboBio Co., Ltd. BNLCL2 cells were transfected with miR-214 mimic, mimic control, Bax plasmid, control-plasmid or miR-214 mimic + Bax plasmid using Lipofectamine ${ }^{\circledR} 2000$ (Invitrogen; Thermo Fisher Scientific, Inc.) in accordance with the manufacturer's instructions. Then, $24 \mathrm{~h}$ after cell transfection, the efficiency of transfection was analyzed using reverse transcription-quantitative PCR (RT-qPCR).

Luciferase reporter assay. miRNA.org software (http://www. microrna.org/microrna/getMirnaForm.do; August 2010 Release) was used to predict the potential target of miR-214. To assess the association between miR-214 and Bax, wild-type (WT) and mutant (MUT) 3'-UTR of Bax containing the miR-214 binding sites, were amplified by RT-PCR using a Transcriptor First Strand cDNA Synthesis kit (Roche Diagnostics), incubating for $5 \mathrm{~min}$ at $25^{\circ} \mathrm{C}$ followed by $60 \mathrm{~min}$ at $42^{\circ} \mathrm{C}$, from total RNA preparations extracted from BNLCL2 cells and cloned into the psiCHECKTM-2 vector (Promega Corporation). The following primer sequences were used: Bax forward, 5'-GGACGAACT GGACAGTAACATGG-3' and reverse, 5'-GCAAAGTAGAAA AGGGCGACAAC-3'. Then, 100 ng psiCHECK-2 luciferase reporter plasmids containing WT and MUT 3'-UTR of Bax were co-transfected into BNLCL2 cells with miR-214 mimic (100 nM) or mimic control (100 nM) for $48 \mathrm{~h}$ using Lipofectamine ${ }^{\circledR} 2000$ (Invitrogen; Thermo Fisher Scientific, Inc.). After 48 h, a Dual Luciferase Assay system (Promega Corporation) was used to detect luciferase activity in the transfected cells. Renilla luciferase activity was used as the control.

ALT and AST detection assay. The levels of AST and ALT were detected in the blood of mice to assess liver injury. Blood $(0.1 \mathrm{ml})$ was collected from each mouse to analyze the serum levels of ALT and AST. The samples were centrifuged at $8,000 \mathrm{x}$ for 8 min at $4^{\circ} \mathrm{C}$ and an automatic biochemical analyzer (Hitachi Ltd.) was used to determine the serum ALT and AST levels according to the manufacturer's protocol.

ELISA. Serum levels of TNF- $\alpha$ (Mouse TNF- $\alpha$ ELISA kit; cat no. PT512) and IL-6 (Mouse IL-6 ELISA kit; cat no. PI326) in D-GalN/LPS-treated mice, and those in the supernatant (centrifugation at $500 \mathrm{x} \mathrm{g}$ at $4^{\circ} \mathrm{C}$ for $5 \mathrm{~min}$ ) of BNLCL2 cells were determined by ELISA kits (Beyotime Institute of Biotechnology) according to the manufacturer's instructions.

TUNEL staining. TUNEL staining was performed to determine cellular apoptosis post-D-GalN/LPS challenge using the in situ cell death detection kit (cat no. 11684817910; Roche Diagnostics) following the manufacturer's instructions. Cells in each group were fixed with $4 \%$ paraformaldehyde at room temperature for $15 \mathrm{~min}$, and dewaxed and hydrated liver tissue sections were permeabilized with $0.1 \%$ Triton X-100 solution for $15 \mathrm{~min}$. Then, $50 \mu \mathrm{l}$ TUNEL reaction mixture (Roche Diagnostics) containing terminal deoxynucleotidyl transferase and fluorescein-dUTP was added into the sample. Subsequently, the cells were incubated at $37^{\circ} \mathrm{C}$ in the dark for $60 \mathrm{~min}$, washed three times with PBS solution for $5 \mathrm{~min}$. 
Then, $50 \mu 1$ Converter-POD (containing anti-fluorescein antibody conjugated with horseradish peroxidase; 1:1,000; cat no. 11684817910; Roche Diagnostics) was added on the sample and incubated at $37^{\circ} \mathrm{C}$ for $30 \mathrm{~min}$. Substrate solution were added and incubated at room temperature for $10 \mathrm{~min}$. The sample was mounted under glass coverslip with PBS and then (random five fields of view) analyzed under a light microscope at x20 magnification (Olympus Corporation). Quantification of the percentage of TUNEL-positive cells was performed using Image-Pro Plus software (version 6.0; Media Cybernetics, Inc.).

Flow cytometry analysis. Flow cytometry was used to analyze apoptosis of BNLCL 2 cells. BNLCL 2 cells $\left(2 \times 10^{6}\right.$ cells/well) were seeded in 6-well culture plates and transfected with mimic control, miR-214 mimic or miR-214 mimic + Bax plasmid for $24 \mathrm{~h}$. Then, cells were treated with D-GalN $(1 \mathrm{mg} / \mathrm{ml})$ and TNF- $\alpha(100 \mathrm{ng} / \mathrm{ml})$ at $37^{\circ} \mathrm{C}$ for $36 \mathrm{~h}$. For each sample, BNLCL2 cells $\left(1 \times 10^{6}\right.$ cells $\left./ \mathrm{ml}\right)$ were trypsinized and re-suspended in binding buffer according to the manufacturer's protocol (Sigma-Aldrich; Merck KGaA). Subsequently, $10 \mu \mathrm{l}$ Annexin V-fluorescein isothiocyanate and $5 \mu 1$ propidium iodide (Beyotime Institute of Biotechnology) were added to BNLCL2 cells, which were stained for $30 \mathrm{~min}$ at room temperature in the dark. A FACSCalibur flow cytometer (BD Biosciences) was used to quantify stained cells and data were analyzed by FlowJo software (version 7.6.1; FlowJo LLC).

$R T$ - $q P C R$. Total RNA was isolated from BNLCL2 cells or liver tissues using TRIzol ${ }^{\circledR}$ reagent (Invitrogen; Thermo Fisher Scientific, Inc.) following the manufacturer's protocol. PrimeScript $^{\mathrm{TM}}$ RT reagent kit (cat no. DRR037A; Takara Bio, Inc.) was used to synthesize cDNA. The reaction condition was as follows: $25^{\circ} \mathrm{C}$ for $5 \mathrm{~min}, 42^{\circ} \mathrm{C}$ for $60 \mathrm{~min}$ and $80^{\circ} \mathrm{C}$ for 2 min. RT-qPCR was performed using SYBR Premix Ex Taq II (Takara Bio, Inc.) with a TaqMan 7900 (ABI) RT PCR system (Applied Biosystems; Thermo Fisher Scientific, Inc.). The thermocycling conditions were as follows: Initial denaturation for $5 \mathrm{~min}$ at $95^{\circ} \mathrm{C}$, followed by 40 cycles of $30 \mathrm{sec}$ at $95^{\circ} \mathrm{C}, 30 \mathrm{sec}$ at $60^{\circ} \mathrm{C}$ and $30 \mathrm{sec}$ at $72^{\circ} \mathrm{C}$, and a final extension step at $72^{\circ} \mathrm{C}$ for $10 \mathrm{~min}$. The primer sequences were as follows: miR-214 forward, 5'-AGCATAATACAGCAGGCACAGAC-3' and reverse, 5'-AAAGGTTGTTCTCCACTCTCTCAC-3'; Bax forward, 5'-GCAGAGGATTGCTGATG-3' and reverse: 5'-CTCAGCCCATATTCTTCCAG-3'; TNF- $\alpha$ forward, 5'-CCACCACGCTCTTCTGTCTAC-3' and reverse, 5'-TGG CTACAGGCTTGTCACT-3'; IL-6 forward, 5'-CCACTTCAC AAGTCGGAGGCTTA-3' and reverse, 5'-GCAAGTGCA TCATCGTTGTTCATAC-3'; U6 forward, 5'-CTCGCTTCG GCAGCACA-3' and reverse, 5'-AACGCTTCACGAATTTGC GT-3'; and GAPDH forward, 5'-CCATGGGGAAGGTGA AGGTC-3' and reverse, 5'-GAAGGGGTCATTGATGGC AAC-3'. U6 and GAPDH were used as endogenous control for miRNAs and mRNAs, respectively. Data were measured using the $2^{-\Delta \Delta \mathrm{Cq}}$ method (17).

Western blotting. Total protein was extracted from liver tissue or cells using lysis buffer [50 mM Tris ( $\mathrm{pH} 8.0), 1 \%$ NP-40, $150 \mathrm{mM} \mathrm{NaCl,} \mathrm{0.1 \%} \mathrm{SDS].} \mathrm{Quantitative} \mathrm{analysis} \mathrm{of}$ the protein was performed using a bicinchoninic acid kit
(Pierce; Thermo Fisher Scientific, Inc.). Equal protein quantities (40 $\mu \mathrm{g}$ per lane) were separated by $12 \%$ SDS-PAGE and transferred to a PVDF membrane (EMD Millipore). Subsequently, the membranes were blocked with 5\% skimmed milk for $1 \mathrm{~h}$ at room temperature and probed with anti-Bax (cat no. SAB4502546; 1:1,000, Sigma Aldrich; Merck KGaA), anti-caspase-3 (cat no. 14220; 1:1,000; Cell Signaling Technology, Inc.) or anti-GAPDH (cat no. G9545; 1:2,000; Sigma Aldrich; Merck KGaA) antibodies overnight at $4{ }^{\circ} \mathrm{C}$. After 4 washes in PBST $(0.1 \%$ Tween-20), the membranes were incubated with Horseradish Peroxidase-conjugated Goat Anti-Rabbit IgG H\&L pre-adsorbed (1:2,000; cat. no. ab7090; Abcam) for $2 \mathrm{~h}$ at room temperature. The immunoreactive proteins were detected using ECL reagent (Pierce; Thermo Fisher Scientific, Inc.).

Statistical analysis. Experiments were repeated three times. Statistical analysis was performed using SPSS 17.0 (IBM Corp.). Data are presented as the mean \pm standard deviation. Differences between groups were analyzed by Student's t-test or one-way analysis of variance with a Bonferroni post hoc test. $\mathrm{P}<0.05$ was considered to indicate a statistically significant difference.

\section{Results}

D-GalN/LPS induces acute liver injury in mice. Liver function and/or the extent of damage in the liver tissues were evaluated by serum ALT and AST levels. Mice were sacrificed at various time points $(0,1,3,5,7$ and $9 \mathrm{~h})$ after D-GalN/LPS challenge, and blood was collected for serum ALT and AST analysis. It was identified that serum ALT and AST levels gradually increased over the $9 \mathrm{~h}$ post-D-GalN/LPS stimulation, peaking at $7 \mathrm{~h}$, compared with the control group, suggesting the presence of liver damage in the mice (Fig. 1A and B). The results also suggested that the maximum level of liver damage was reached at $7 \mathrm{~h}$ after D-GalN/LPS stimulation, thus mice at $7 \mathrm{~h}$ after D-GalN/LPS stimulation was selected in subsequent experimentations.

IL-6 and TNF- $\alpha$ are involved in hepatocyte apoptosis and regeneration $(18,19)$. Therefore, the levels of IL-6 and TNF- $\alpha$ in the serum of mice were measured after D-GalN/LPS treatment. It was demonstrated that the levels of IL- 6 and TNF- $\alpha$ were significantly increased at $7 \mathrm{~h}$ post-D-GalN/LPS challenge compared with the saline-treated group (Fig. 1C and D).

D-GalN/LPS stimulates hepatocyte apoptosis in mice. Apoptosis of hepatocytes was assessed by TUNEL staining, and it was identified that the percentage of apoptotic cells was significantly increased at $7 \mathrm{~h}$ post D-GalN/LPS challenge compared with the saline-treated group (Fig. 2A). Furthermore, the expression of the apoptotic-associated protein caspase-3 was analyzed, and D-GalN/LPS-challenged mice exhibited increased caspase-3 protein expression at $7 \mathrm{~h}$ post D-GalN/LPS challenge compared with saline-treated mice (Fig. 2B).

miR-214 is downregulated in D-GalN/LPS-stimulated mice. The RT-qPCR results demonstrated that the mRNA expression 
A

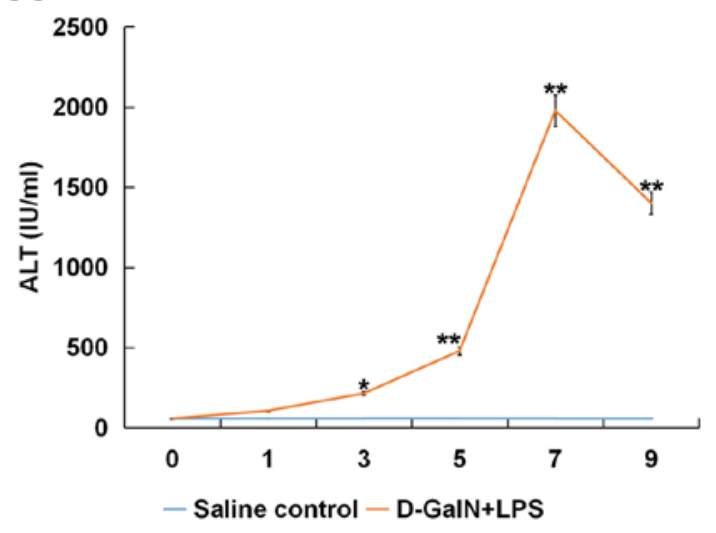

C

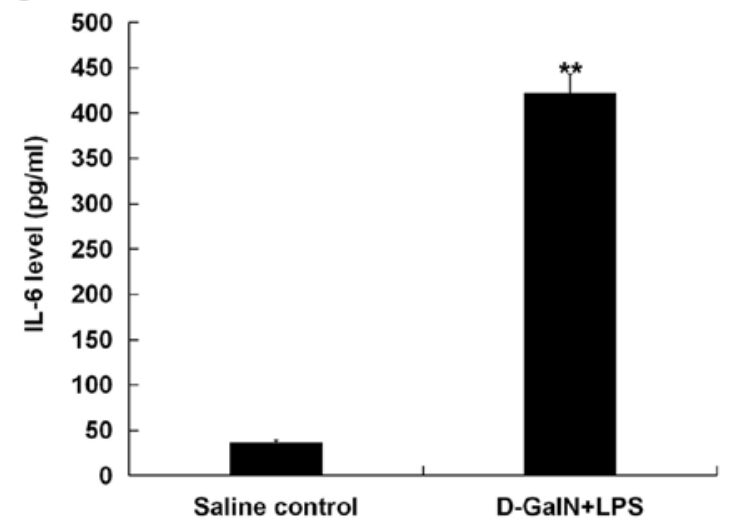

B

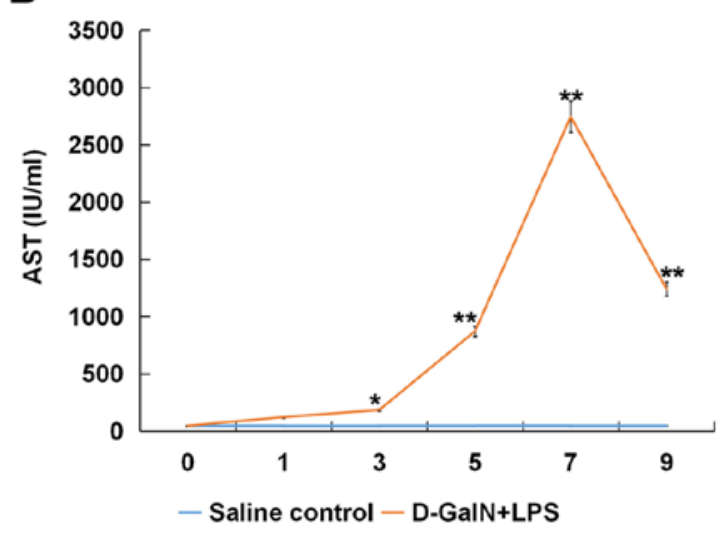

D

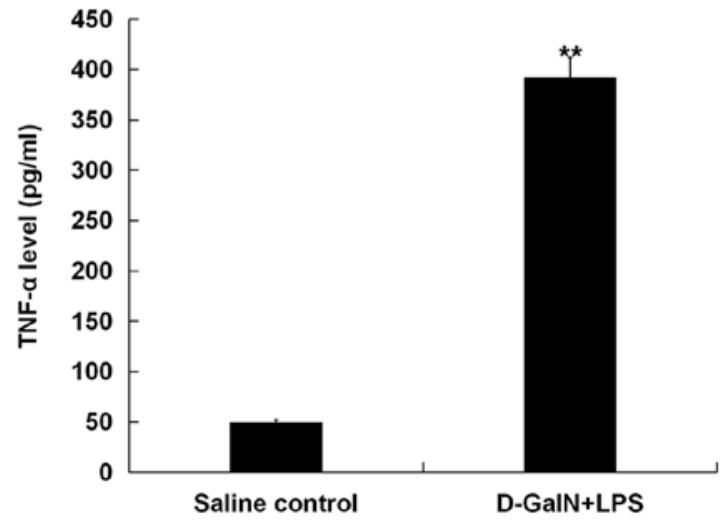

Figure 1. D-GalN/LPS induces acute liver injury in mice. Mice were sacrificed at various time points $(0,1,3,5,7$ and 9 h) after D-GalN/LPS challenge, and blood was collected for serum ALT and AST analysis. Serum (A) ALT and (B) AST release increased gradually and peaked at $7 \mathrm{~h}$ post-D-GalN/LPS challenge, compared with the saline injection group. Serum concentrations of (C) IL-6 and (D) TNF- $\alpha$ in D-GalN/LPS-treated mice increased significantly at $7 \mathrm{~h}$ after D-GalN/LPS treatment compared with the saline-treated group. Data are presented as the mean \pm standard deviation. ${ }^{*} \mathrm{P}<0.05$ and ${ }^{* *} \mathrm{P}<0.01$ vs. saline control group. D-GalN, D-galactosamine; LPS, lipopolysaccharide; TNF- $\alpha$, tumor necrosis factor- $\alpha$; IL, interleukin; AST, aspartate aminotransferase; ALT, alanine aminotransferase.

A

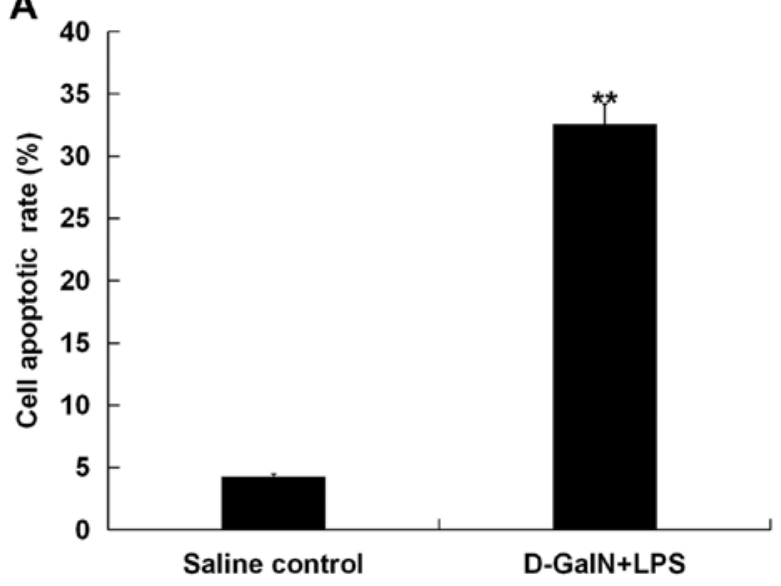

B

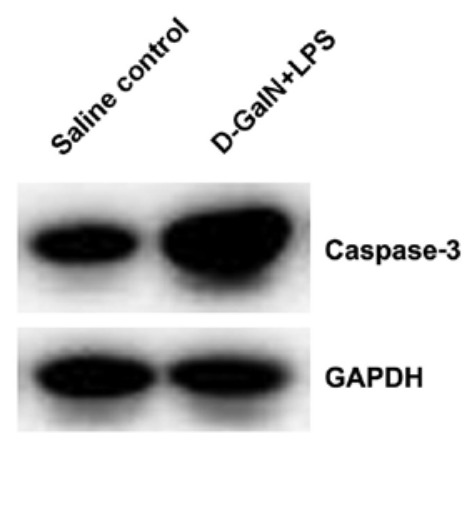

Figure 2. Increased hepatocyte apoptosis in D-GalN/LPS-stimulated mice. (A) Apoptosis of hepatocytes in the mouse liver tissues was assessed by TUNEL staining at $7 \mathrm{~h}$ after D-GalN/LPS treatment, and the apoptotic rate of hepatocytes was calculated. (B) Protein expression of caspase-3 in the mice liver tissues was detected using western blotting at $7 \mathrm{~h}$ after D-GalN/LPS treatment. Data are presented as the mean \pm standard deviation. ${ }^{* *} \mathrm{P}<0.01 \mathrm{vs}$. saline control group. D-GalN, D-galactosamine; LPS, lipopolysaccharide.

of miR-214 was significantly downregulated in the liver tissue of D-GalN/LPS-stimulated mice compared with the saline control group (Fig. 3A).
Bax is a target gene of miR-214 and is upregulated in $D$-GalN/LPS-stimulated mice. miRNA.org software predicted the binding sites between Bax and miR-214 (Fig. 3B), and a 
A

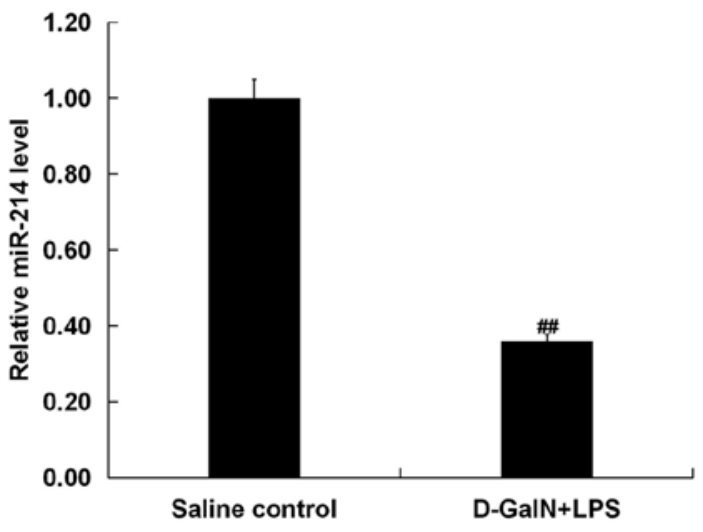

D

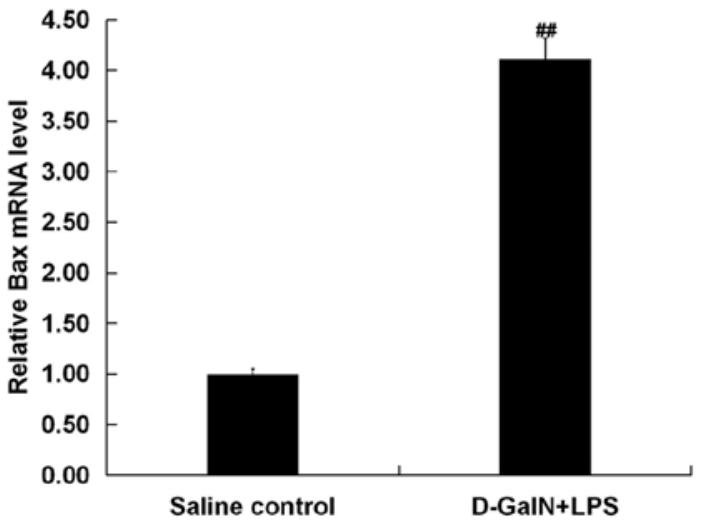

B

3' ugacggacagacacGGACGACa 5' hsa-miR-214 |||||||

5' eccgauucaucuacCCUGCUGa 3' BAX

C

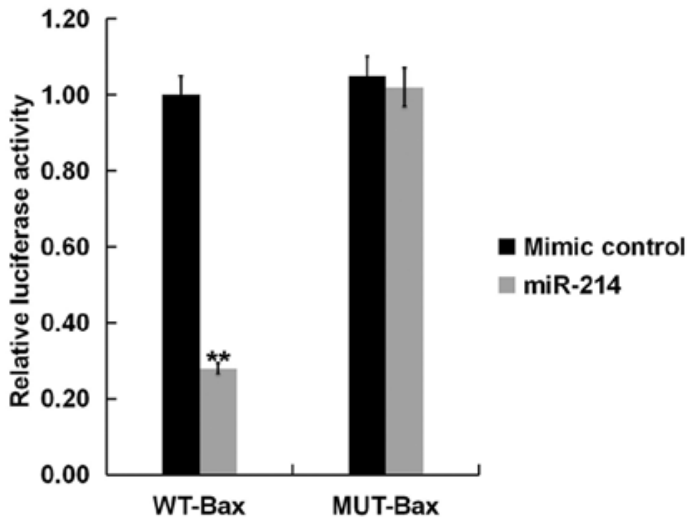

E

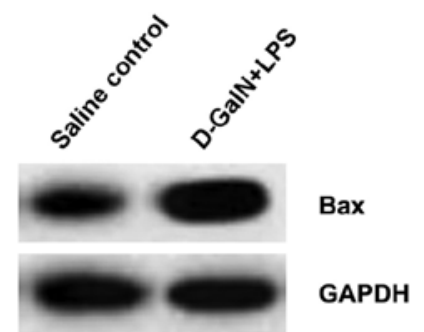

Figure 3. miR-214 and Bax expression in D-GalN/LPS-stimulated mice. (A) mRNA expression of miR-214 in the liver tissues of mice was detected using RT-qPCR. (B) Binding sites between miR-214 and Bax. (C) Luciferase reporter assay was conducted to identify the binding sites between miR-214 and Bax. (D) mRNA and (E) protein expression levels of Bax in the liver tissues of mice were detected using RT-qPCR and western blotting at $7 \mathrm{~h}$ after D-GalN/LPS treatment. Data are presented as the mean \pm standard deviation. ${ }^{* *} \mathrm{P}<0.01$ vs. mimic control. ${ }^{\# \#} \mathrm{P}<0.01$ vs. saline control group. $\mathrm{D}-\mathrm{GalN}, \mathrm{D}$-galactosamine; LPS, lipopolysaccharide; RT-qPCR, reverse transcription-quantitative polymerase chain reaction; miR, microRNA; WT, wild-type; MUT, mutant.

dual luciferase reporter assay was conducted to examine the identified miR-214 binding sites in the 3'-UTR of Bax. The results indicated that treatment with the miR-214 mimic decreased the relative luciferase activity of Bax-WT, but had no effect on Bax-MUT, compared with the mimic control group (Fig. 3C). Therefore, it was speculated that Bax may be a target gene of miR-214. In addition, Bax mRNA (Fig. 3D) and protein (Fig. 3E) expression levels were significantly increased in the liver tissue of mice at $7 \mathrm{~h}$ post D-GalN/LPS stimulation compared with the saline control group.

miR-214 is downregulated and Bax is upregulated in $D$-GalN/TNF- $\alpha$-stimulated hepatocytes. Subsequent experiments were conducted in an in vitro model of BNLCL2 cells stimulated by D-GalN and TNF- $\alpha$. The expression of miR-214 was first detected in BNLCL2 cells treated with (model) or without (control) D-GalN/TNF- $\alpha$. The results indicated that, compared with the control group, miR-214 expression was significantly decreased in D-GalN/TNF- $\alpha$-treated BNLCL2 cells (Fig. 4A). In addition, compared with the control group, Bax was significantly increased in D-GalN/TNF- $\alpha$-treated BNLCL2 cells at both the mRNA (Fig. 4B) and protein expression levels (Fig. 4C).
miR-214 mimic inhibit cell apoptosis and inflammation in $D$-GalN/TNF- $\alpha$-stimulated hepatocytes. To investigate the regulatory role of miR-214 in D-GalN/TNF- $\alpha$-stimulated hepatocytes, BNLCL2 cells were transfected with mimic control, miR-214 mimic or miR-214 mimic + Bax plasmid for $24 \mathrm{~h}$; subsequently, cells were treated with D-GalN $(1 \mathrm{mg} / \mathrm{ml})$ and TNF- $\alpha(100 \mathrm{ng} / \mathrm{ml})$ for $36 \mathrm{~h}$. Transfection efficiencies were detected by RT-qPCR, and it was identified that miR-214 mimic transfection caused a significant increase in miR-214 mRNA expression (Fig. 5A), and that Bax plasmid transfection caused a significant increase in Bax (Fig. 5B) in BNLCL2 cells. In addition, miR-214 overexpression resulted in the downregulation of the mRNA and protein expression levels of Bax in BNLCL2 cells, and this downregulation was reversed by Bax plasmid transfection (Fig. 5C and D).

Furthermore, flow cytometry results demonstrated that D-GalN/TNF- $\alpha$ treatment significantly enhanced BNLCL2 cell apoptosis compared with the control group. Moreover, compared with the D-GalN/TNF- $\alpha$ treatment alone group, the results indicated that the miR-214 mimic significantly decreased BNLCL2 cell apoptosis, which was reversed by Bax plasmid transfection (Fig. 6A and B). 
A

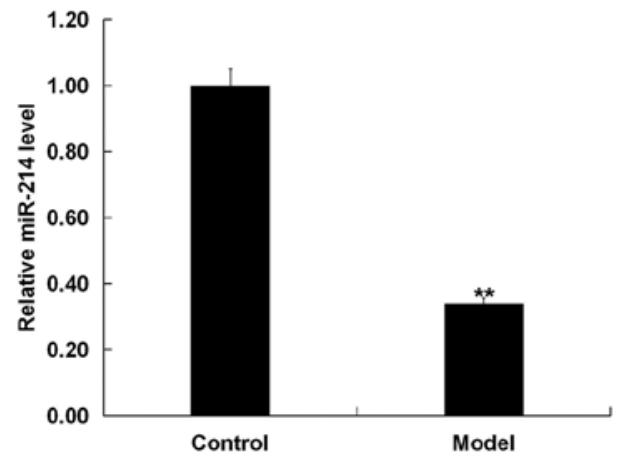

B

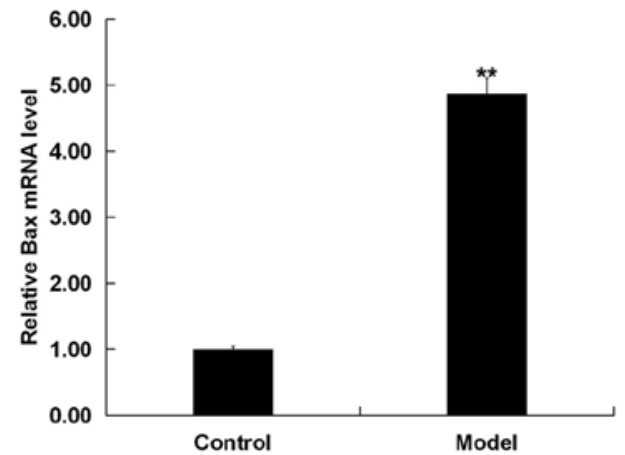

C

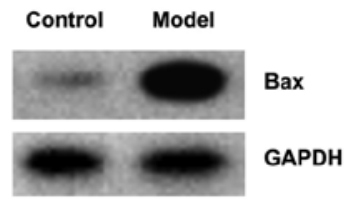

Figure 4. miR-214 and Bax in D-GalN/TNF- $\alpha$-stimulated hepatocytes. (A) Expression of miR-214 in hepatocytes treated with (model) or without (control) D-GalN/TNF- $\alpha$ was detected using RT-qPCR. (B) mRNA and (C) protein expression levels of Bax in hepatocytes treated with (model) or without (control) D-GalN/TNF- $\alpha$ were detected using RT-qPCR and western blotting. Data are presented as the mean \pm standard deviation. ${ }^{* *} \mathrm{P}<0.01$ vs. control. D-GalN, D-galactosamine; LPS, lipopolysaccharide; TNF- $\alpha$, tumor necrosis factor- $\alpha$; RT-qPCR, reverse transcription-quantitative polymerase chain reaction; miR, microRNA.

A

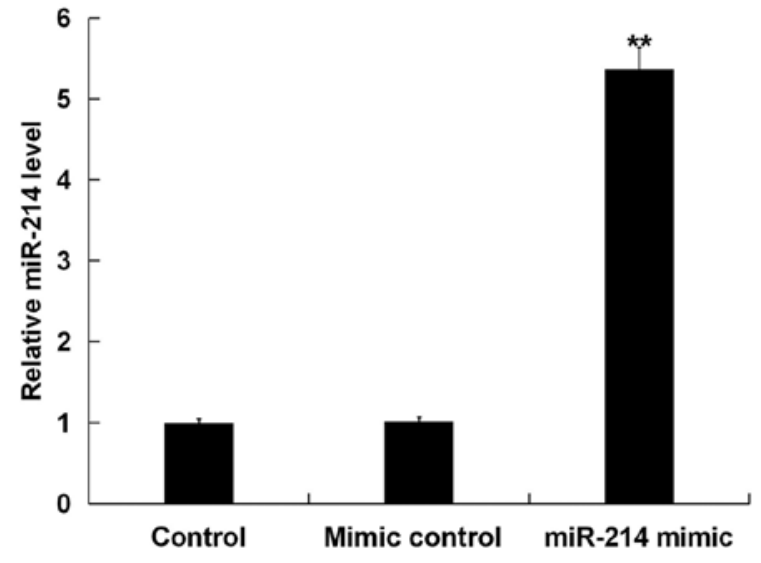

\section{C}

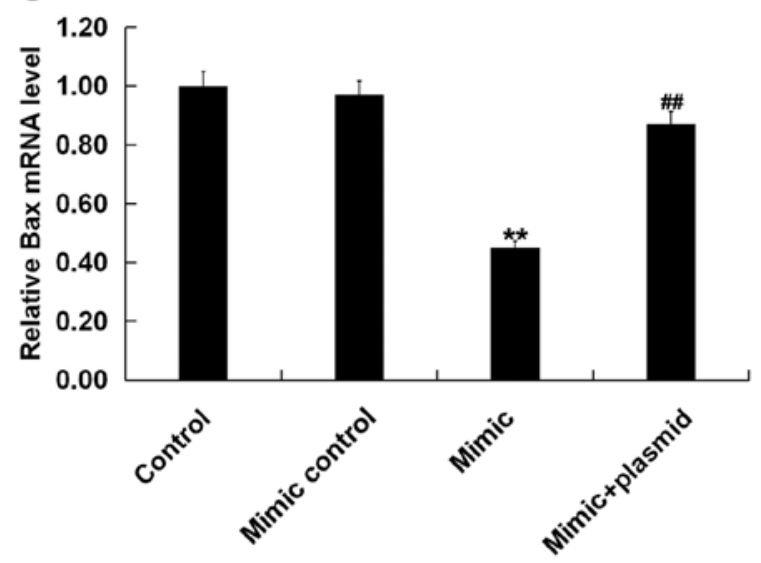

B

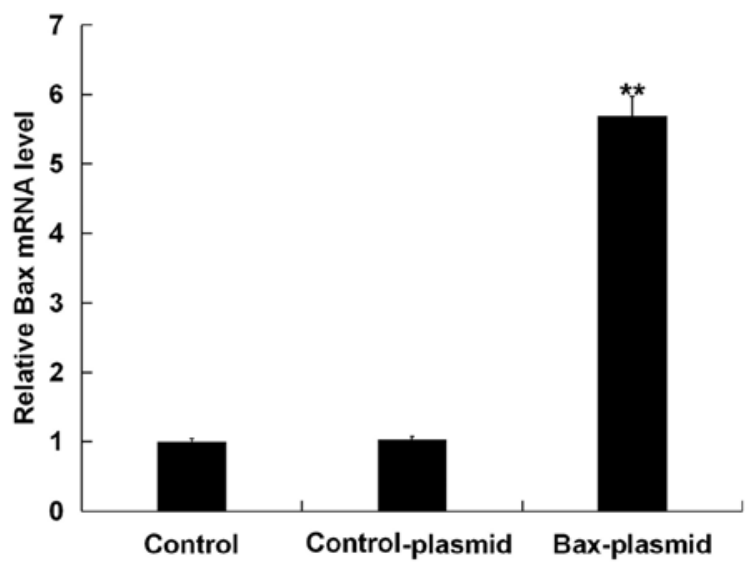

D

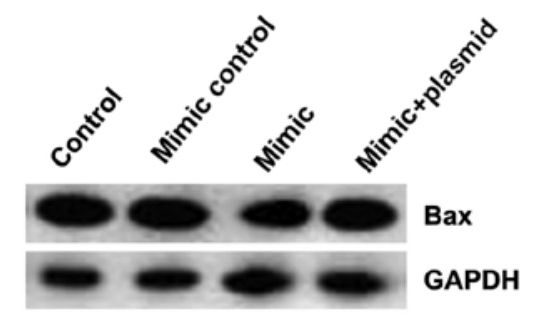

Figure 5. miR-214 negatively regulates Bax expression in hepatocytes. (A) mRNA expression of miR-214 in BNLCL2 cells was measured using RT-qPCR. (B) mRNA expression of Bax in BNLCL2 cells was determined using RT-qPCR. (C) mRNA and (D) protein expression levels of Bax in BNLCL2 cells were detected using RT-qPCR and western blotting. Data are presented as the mean \pm standard deviation. ${ }^{* *} \mathrm{P}<0.01 \mathrm{vs}$. control. ${ }^{\# \#} \mathrm{P}<0.01 \mathrm{vs}$. mimic. RT-qPCR, reverse transcription-quantitative polymerase chain reaction; miR, microRNA.

In concordance with the in vivo results, the mRNA and protein expression levels of TNF- $\alpha$ (Fig. 7A and C) and IL-6 (Fig. 7B and D) in BNLCL2 cells post-D-GalN/TNF- $\alpha$ challenge were significantly increased compared with the control group. Furthermore, the results suggested that miR-214 mimic transfection significantly decreased the mRNA expression and 
A

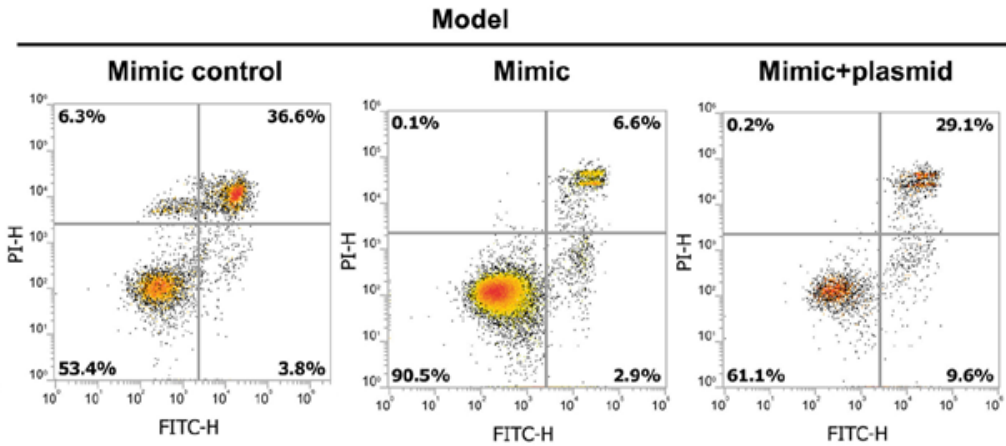

B

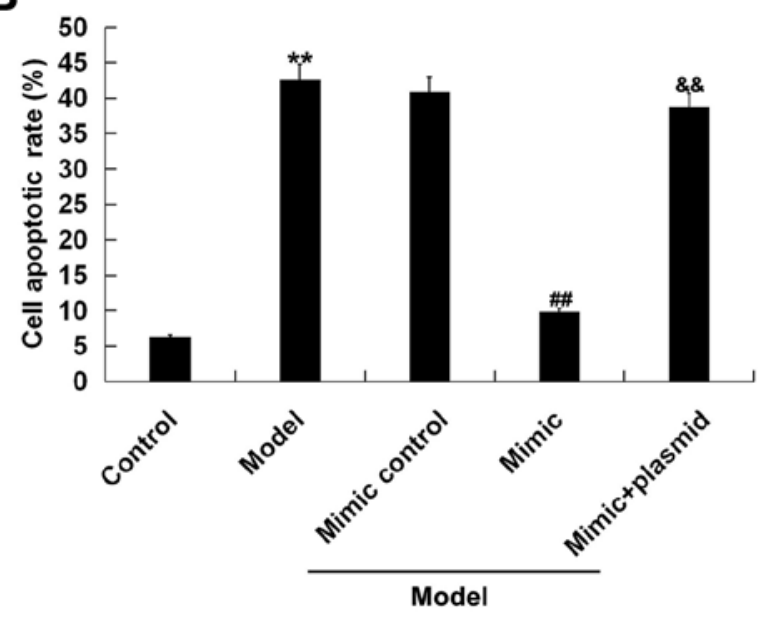

Figure 6. miR-214 inhibits cell apoptosis in D-GalN/TNF- $\alpha$-stimulated hepatocytes. BNLCL2 cells were transfected with mimic control, miR-214 mimic or miR-214 mimic + Bax plasmid for $24 \mathrm{~h}$, then cells were treated with D-GalN (1 mg/ml) and TNF- $\alpha$ (100 ng/ml) for $36 \mathrm{~h}$. (A) BNLCL2 cell apoptosis was analyzed by flow cytometry and (B) cell apoptosis rate was calculated. Data are presented as the mean \pm standard deviation. ${ }^{* *} \mathrm{P}<0.01$ vs. control. ${ }^{\# \#} \mathrm{P}<0.01$ vs. model. ${ }^{\& \&} \mathrm{P}<0.01$ vs. mimic. D-GalN, D-galactosamine; LPS, lipopolysaccharide; TNF- $\alpha$, tumor necrosis factor- $\alpha$; FITC, fluorescein isothiocyanate; PI, propidium iodide.

protein levels of TNF- $\alpha$ and IL- 6 in BNLCL2 cells stimulated by $\mathrm{D}-\mathrm{GalN} / \mathrm{TNF}-\alpha$, and all of these changes were reversed by the Bax plasmid.

\section{Discussion}

ALF is a condition associated with high mortality (1), but its underlying pathological mechanism remains largely unknown. Therefore, the development of novel prognostic biomarkers and therapeutic targets for ALF is crucial. miRNAs have been reported to regulate various aspects of hepatic function, including cell proliferation, metabolism and viral infection (20). The present results demonstrated that miR-214 was downregulated and Bax was upregulated in a D-GalN/LPS-induced murine ALF model. In addition, the results indicated that miR-214 ameliorated ALF via the regulation of Bax expression.

D-GalN/LPS has been widely used to induce hepatic damage, accompanied by changes in hepatic apoptosis and necrosis, which are similar to the changes observed in human viral hepatitis (21). In the present study, D-GalN and LPS, which are well known for ALF induction (15,21-23), were used to induce an experimental acute liver injury model in mice. Consistent with previous results (15), the present results suggested that D-GalN/LPS significantly increased the levels of serum AST and ALT, enhanced the expression levels of the pro-inflammatory factors TNF- $\alpha$ and IL-6, and promoted hepatocyte apoptosis. Moreover, these results indicated that the D-GalN/LPS-induced ALF model was successfully established. In addition, miR-214 was identified to be significantly decreased in the liver tissue of D-GalN/LPS-induced mice. However, in the present study, groups of mice treated with only D-GalN or only LPS were not conducted, which may be a limitation, and thus further examination in future studies is required. Moreover, Bax was identified to be a direct target of miR-214 in BNLCL2 cells. However, the association between miR-214 and Bax in other hepatocyte cell lines was not investigated in the present study. Therefore, this is a limitation of the present study, and must be elucidated in the future.

ALF is characterized by extensive hepatocyte apoptosis and necrosis (24). Furthermore, 2 major mechanisms of cell death, namely the death receptor pathway and the mitochondrial pathway, are involved in the progression of ALF, in which TNF serves a crucial role (25). The caspase family is a key contributor to cell apoptosis, and it has been reported that caspase-3 is significantly activated in the ALF model (26). In addition, apoptotic-associated proteins tightly control cell apoptosis (27). For example, members of the Bcl-2 family interact with members of the Bax subfamily to induce apoptosis signals and cause apoptosis (28). In a GalN/LPS-treated mouse model, Bcl-2 expression was significantly decreased, while Bax expression was increased (29). Consistent with 
A

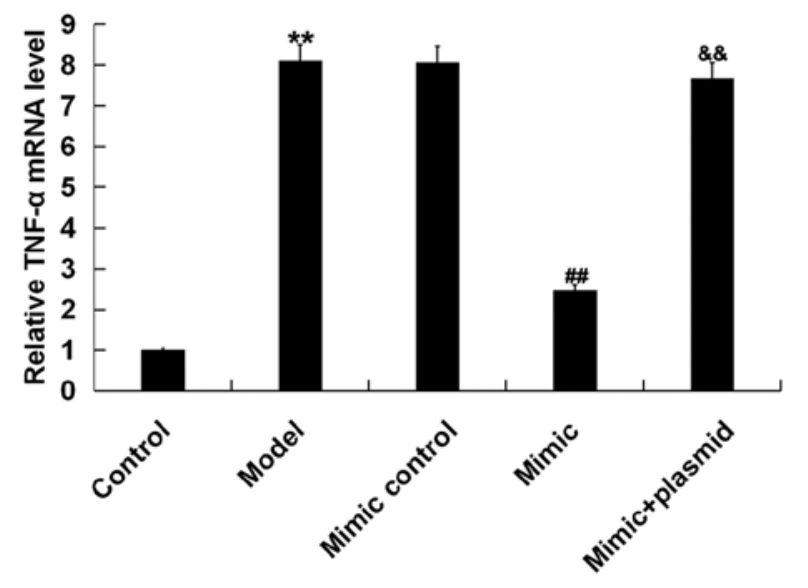

C

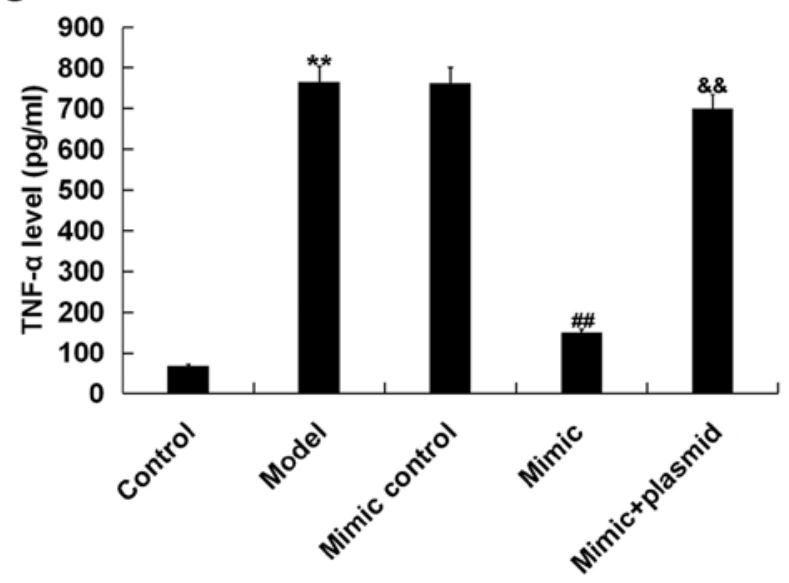

B

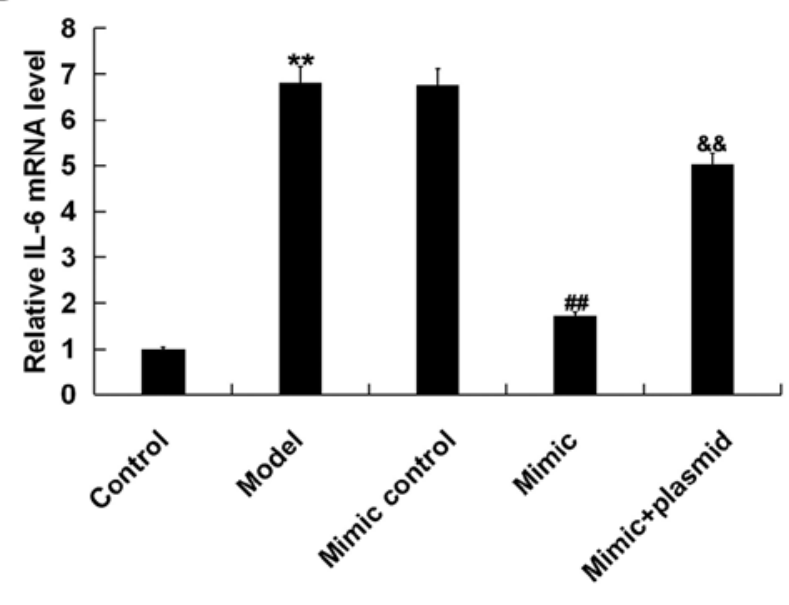

D

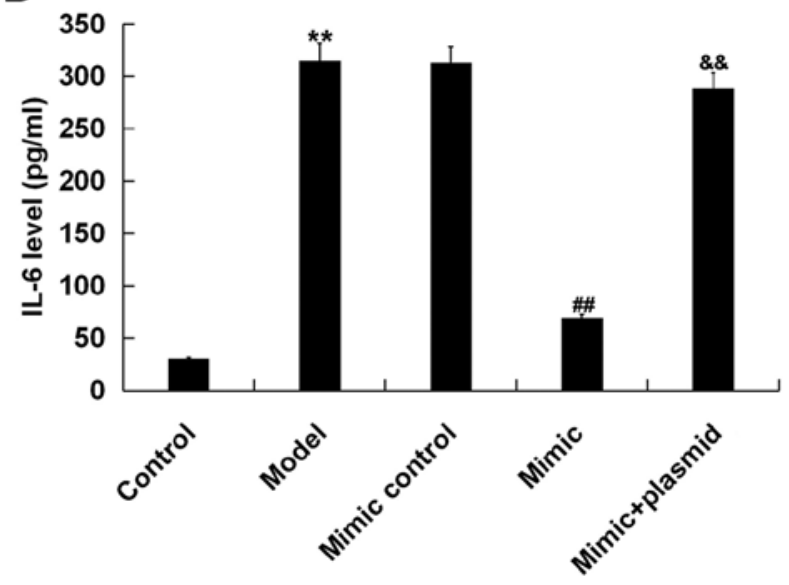

Figure 7. miR-214 decreases TNF- $\alpha$ and IL-6 levels in D-GalN/TNF- $\alpha$-stimulated hepatocytes. BNLCL2 cells were transfected with mimic control, miR-214 mimic or miR-214 mimic + Bax plasmid for $24 \mathrm{~h}$, then cells were treated with D-GalN (1 mg/ml) and TNF- $\alpha$ (100 ng/ml) for $36 \mathrm{~h}$. (A) TNF- $\alpha$ and (B) IL-6 mRNA expression levels were measured by reverse transcription-quantitative polymerase chain reaction. Release of (C) TNF- $\alpha$ and (D) IL-6 in the culture medium of BNLCL2 cells was detected using ELISA. Data are presented as the mean \pm standard deviation. ${ }^{* *} \mathrm{P}<0.01$ vs. control. ${ }^{\# \#} \mathrm{P}<0.01$ vs. model; ${ }^{\&} \mathrm{P}<0.01$ vs. mimic. D-GalN, D-galactosamine; LPS, lipopolysaccharide; TNF- $\alpha$, tumor necrosis factor- $\alpha$; IL, interleukin; miR, microRNA.

previous studies $(15,21-23)$, the present results demonstrated that D-GalN/LPS treatment significantly increased hepatocyte apoptosis and increased the expression Bax in vivo and in vitro.

Ameliorated hepatocyte apoptosis has been shown to be a key step in the mitigation of D-GalN/LPS-induced ALF (30). Previous studies have also reported that miRNAs can regulate the expression of pro-apoptotic and anti-apoptotic genes (31). Moreover, it has been reported that miR-15b and miR-16 negatively adjust TNF- $\alpha$-mediated liver cell apoptosis via $\mathrm{Bcl} 2$ in severe liver failure (32). In addition, miR-24 regulates the key apoptotic gene BCL2-like protein 11 during ALF (15). The present results indicated that miR-214 expression was downregulated in the liver tissues from mice stimulated with D-GalN/LPS. It has also been reported that miR-214 serves a suppressive role on extrinsic cell death pathways, such as necrosis and autophagy (33). A previous study indicated that miR-214 improves acute kidney injury in vivo by inhibiting apoptosis (34). miR-214 has also been demonstrated to protect cells from hypoxia/reoxygenation-induced damage and attenuates ischemia/reperfusion (I/R)-induced myocardial injury via suppression of PTEN and Bcl-2 homology domain 3 (BH3)-only Pro-Protein expression levels, leading to decreases in I/R-induced myocardial apoptosis (35). The results of the present study demonstrated that miR-214 ameliorated D-GalN/TNF- $\alpha$-induced cell apoptosis via targeting Bax. However, the specific association between these miRNAs, including miR-214, miR-24, miR-15b and miR-16, in liver injury requires further research.

In conclusion, the present results suggested that miR-214 was downregulated in a D-GalN/LPS-induced murine ALF model and in D-GalN/TNF- $\alpha$-stimulated hepatocytes. Moreover, it was identified that miR-214 ameliorated D-GalN/TNF- $\alpha$-induced inflammation and apoptosis in hepatocytes via targeting Bax. Therefore, it was hypothesized that miR-214 may serve as a novel therapeutic strategy for ALF treatment. However, this is only a preliminary study on the role of miR-214 in ALF, and the present study had limitations; therefore, additional in-depth research is required to establish the role of miR-214 in ALF. For example, the effects of miR-214, on factors other than cell apoptosis, such as hepatocyte function and stress markers in hepatocytes, should be subsequently investigated. In addition, the present study focused on the effect of miR-214 on normal murine embryonic liver cells. However, due to the interspecies variation in hepatic 
responses, it is necessary to study the effect of miR-214 on human hepatocytes; this was also a limitation of the present study, which will be addressed in future research.

\section{Acknowledgements}

Not applicable.

\section{Funding}

The present study was supported by the National S\&T Major Project (grant no. 2017ZX10203201-002-002).

\section{Availability of data and materials}

The datasets used and/or analyzed during the current study are available from the corresponding author on reasonable request.

\section{Authors' contributions}

SW contributed to study design, data collection, statistical analysis, data interpretation and manuscript preparation. $\mathrm{XH}$, WS, LC, YH, YW, EL, AQ and WZ contributed to data collection and statistical analysis. JG contributed to data collection, statistical analysis and manuscript preparation. All authors read and approved the final manuscript.

\section{Ethics approval and consent to participate}

All animal care and animal experimental protocols were carried out strictly according to the recommendations in the Guide for the Care and Use of Laboratory Animals by the National Institutes of Health and the Animal Ethics Committee of The First Affiliated Hospital of Suzhou University. The present study was approved by the Animal Ethics Committee of The First Affiliated Hospital of Suzhou University.

\section{Patient consent for publication}

Not applicable.

\section{Competing interests}

The authors declare that they have no competing interests.

\section{References}

1. Grek A and Arasi L: Acute liver failure. AACN Adv Crit Care 27: 420-429, 2016.

2. Bernal W and Wendon J: Acute liver failure. N Engl J Med 369: 2525-2534, 2013.

3. Li X, Gou C, Yang H, Qiu J, Gu T and Wen T: Echinacoside ameliorates D-galactosamine plus lipopolysaccharide-induced acute liver injury in mice via inhibition of apoptosis and inflammation. Scand J Gastroenterol 49: 993-1000, 2014.

4. Dong V, Nanchal R and Karvellas CJ: Pathophysiology of acute liver failure. Nutr Clin Pract 35: 24-29, 2020.

5. Wu Z, Han M, Chen T, Yan W and Ning Q: Acute liver failure: Mechanisms of immune-mediated liver injury. Liver Int 30: 782-794, 2010.

6. Ambros V: MicroRNA pathways in flies and worms: Growth, death, fat, stress, and timing. Cell 113: 673-676, 2003.
7. Antoine DJ, Dear JW, Lewis PS, Platt V, Coyle J, Masson M, Thanacoody RH, Gray AJ, Webb DJ, Moggs JG, et al: Mechanistic biomarkers provide early and sensitive detection of acetaminophen-induced acute liver injury at first presentation to hospital. Hepatology 58: 777-787, 2013.

8. Song G, Sharma AD, Roll GR, Ng R, Lee AY, Blelloch RH, Frandsen NM and Willenbring $\mathrm{H}$ : MicroRNAs control hepatocyte proliferation during liver regeneration. Hepatology 51: $1735-1743,2010$

9. Szabo $G$ and Bala S: MicroRNAs in liver disease. Nat Rev Gastroenterol Hepatol 10: 542-552, 2013.

10. Wang XW, Heegaard NH and Orum H: MicroRNAs in liver disease. Gastroenterology 142: 1431-1443, 2012.

11. Wang D, Sun X, Wei Y, Liang H, Yuan M, Jin F, Chen X, Liu Y, Zhang CY, Li L and Zen K: Nuclear miR-122 directly regulates the biogenesis of cell survival oncomiR miR-21 at the posttranscriptional level. Nucleic Acids Res 46: 2012-2029, 2018.

12. Bandiera S, Pfeffer S, Baumert TF and Zeisel MB: miR-122-a key factor and therapeutic target in liver disease. J Hepatol 62: 448-457, 2015

13. Yang D, Yuan Q, Balakrishnan A, Bantel H, Klusmann JH, Manns MP, Ott M, Cantz T and Sharma AD: MicroRNA-125b-5p mimic inhibits acute liver failure. Nat Commun 7: 11916, 2016.

14. Izawa T, Horiuchi T, Atarashi M, Kuwamura M and Yamate J: Anti-fibrotic role of miR-214 in thioacetamide-induced liver cirrhosis in rats. Toxicol Pathol 43: 844-851, 2015.

15. Feng Z, Li Z, Zhu D, Ling W, Zheng L, Pu L and Kong L: Mir-24 regulates hepatocyte apoptosis via BIM during acute liver failure. Am J Transl Res 9: 4925-4935, 2017.

16. Lu TX and Rothenberg ME: MicroRNA. J Allergy Clin Immunol 141: 1202-1207, 2018.

17. Livak KJ and Schmittgen TD: Analysis of relative gene expression data using real-time quantitative PCR and the 2(-Delta Delta C (T)) method. Methods 25: 402-408, 2001.

18. Kuhla A, Eipel C, Siebert N, Abshagen K, Menger MD and Vollmar B: Hepatocellular apoptosis is mediated by TNFalpha-dependent Fas/FasLigand cytotoxicity in a murine model of acute liver failure. Apoptosis 13: 1427-1438, 2008.

19. Wan J, Benkdane M, Alons E, Lotersztajn S and Pavoine C: M2 kupffer cells promote hepatocyte senescence: An IL-6-dependent protective mechanism against alcoholic liver disease. Am J Pathol 184: 1763-1772, 2014.

20. Kerr TA, Korenblat KM and Davidson NO: MicroRNAs and liver disease. Transl Res 157: 241-252, 2011.

21. Wen J, Lin H, Zhao M, Tao L, Yang Y, Xu X, Jia A, Zhang J and Weng D: Piceatannol attenuates D-GalN/LPS-induced hepatoxicity in mice: Involvement of ER stress, inflammation and oxidative stress. Int Immunopharmacol 64: 131-139, 2018.

22. Bian X, Liu X, Liu J, Zhao Y, Li H, Zhang L, Li P and Gao Y: Hepatoprotective effect of chiisanoside from Acanthopanax sessiliflorus against LPS/D-GalN-induced acute liver injury by inhibiting $\mathrm{NF}-\kappa \mathrm{B}$ and activating Nrf2/HO-1 signaling pathways. J Sci Food Agric 99: 3283-3290, 2019.

23. Wang H, Chen L, Zhang X, Xu L, Xie B, Shi H, Duan Z, Zhang $H$ and Ren F: Kaempferol protects mice from d-GalN/LPS-induced acute liver failure by regulating the ER stress-Grp78-CHOP signaling pathway. Biomed Pharmacother 111: 468-475, 2019.

24. Rutherford A and Chung RT: Acute liver failure: Mechanisms of hepatocyte injury and regeneration. Semin Liver Dis 28: 167-174, 2008.

25. Schwabe RF and Luedde T: Apoptosis and necroptosis in the liver: A matter of life and death. Nat Rev Gastroenterol Hepatol 15: 738-752, 2018.

26. Shirozu K, Hirai S, Tanaka T, Hisaka S, Kaneki M and Ichinose F: Farnesyltransferase inhibitor, tipifarnib, prevents galactosamine/lipopolysaccharide-induced acute liver failure. Shock 42: 570-577, 2014.

27. Gómez-Fernández JC: Functions of the C-terminal domains of apoptosis-related proteins of the Bcl-2 family. Chem Phys Lipids 183: 77-90, 2014

28. Zhang RZ, Qiu H, Wang N, Long FL and Mao DW: Effect of rheum palmatum $\mathrm{L}$. on $\mathrm{NF}-\kappa \mathrm{B}$ signaling pathway of mice with acute liver failure. Asian Pac J Trop Med 8: 841-847, 2015.

29. Xu L, Zheng X, Wang Y, Fan Q, Zhang M, Li R, Ye J, $\mathrm{Wu} \mathrm{X}, \mathrm{Zhao} \mathrm{W}$ and Zhang Y: Berberine protects acute liver failure in mice through inhibiting inflammation and mitochondria-dependent apoptosis. Eur J Pharmacol 819: $161-168,2018$ 
30. Nakama T, Hirono S, Moriuchi A, Hasuike S, Nagata K, Hori T, Ido A, Hayashi $\mathrm{K}$ and Tsubouchi $\mathrm{H}$ : Etoposide prevents apoptosis in mouse liver with D-galactosamine/lipopolysaccharide-induced fulminant hepatic failure resulting in reduction of lethality. Hepatology 33: 1441-1450, 2001.

31. Garofalo M, Condorelli GL, Croce CM and Condorelli G MicroRNAs as regulators of death receptors signaling. Cell Death Differ 17: 200-208, 2010.

32. An F, Gong B, Wang H, Yu D, Zhao G, Lin L, Tang W, Yu H, Bao S and Xie Q: miR-15b and miR-16 regulate TNF mediated hepatocyte apoptosis via BCL2 in acute liver failure. Apoptosis 17: 702-716, 2012.

33. Ghaderi S, Alidadiani N, SoleimaniRad J, Heidari HR, Dilaver N, Heim C, Ramsperger-Gleixner M, Baradaran B and Weyand M: DJ1 and microRNA-214 act synergistically to rescue myoblast cells after ischemia/reperfusion injury. J Cell Biochem 119: 7192-7203, 2018
34. Zhu X, Li W and Li H: miR-214 ameliorates acute kidney injury via targeting DKK3 and activating of Wnt/ $\beta$-catenin signaling pathway. Biol Res 51: 31, 2018.

35. Wang X, Ha T, Hu Y, Lu C, Liu L, Zhang X, Kao R, Kalbfleisch J, Williams D and Li C: MicroRNA-214 protects against hypoxia/reoxygenation induced cell damage and myocardial ischemia/reperfusion injury via suppression of PTEN and Bim1 expression. Oncotarget 7: 86926-86936, 2016.

This work is licensed under a Creative Commons Attribution-NonCommercial-NoDerivatives 4.0 International (CC BY-NC-ND 4.0) License. 